\title{
Apoptosis and karyogamy in syncytia induced by the HIV-1-envelope glycoprotein complex
}

\begin{abstract}
Dear Editor,
One of the several mechanisms accounting for HIV-1induced lymphodepletion resides in the capacity of the envelope glycoprotein complex (Env) expressed on HIV-1 infected cells to interact with CD4 and a suitable coreceptor (CXCR4 or CCR5) expressed on non-infected cells, thereby triggering cell fusion. ${ }^{1}$ The vast majority of syncytium-inducing HIV-1 variants employs CXCR4 as a coreceptor, and a strong correlation between $\mathrm{CD}^{+} \mathrm{T}$ cell decline and infection by syncytium-induced HIV-1 variants has been established. Formation of syncytia in vitro is generally followed by cell death, either by apoptosis or necrosis, depending on the cell types engaged in the process. ${ }^{2,3}$ In vivo, in lymphoid tissues from AIDS patients, syncytium formation is accompanied by overexpression of tissue transglutaminase, a marker of apoptosis. ${ }^{4}$
\end{abstract}

In a model culture system of syncytium-dependent cell death, HeLa cells stably transfected with a lymphotropic HIV-1 Env gene (HeLa Env) were fused by co-culture with CD4/CXCR4-expressing HeLa cells (Hela CD4). ${ }^{5,6}$ As an internal control, HeLa cell expressing a monotropic HIV-1 envelope (ADA, that does not interact with the CXCR4 co-receptor expressed on HeLa CD4) failed to form syncytia with HeLa CD4 cells and did not undergo apoptosis $^{7}$ (Figure 1A). Fusion events were monitored by means of two stable, non-toxic CellTracker fluorescent dies with which HeLa Env (CellTracker Green) or HeLa CD4 cells (CellTracker Orange) were pre-incubated. After $24 \mathrm{~h}$ of co-culture, juxtaposed nuclei from both cell types could be clearly distinguished within a common cytoplasm, the center of syncytia (Figure 1A) whose overall organization roughly recapitulates that of normal cells. ${ }^{1}$ After $48 \mathrm{~h}$, however, nucleoplasm fusion (karyogamy) occurred in $\sim 50 \%$ of syncytia, as detectable by the blending of the two CellTracker dies within the nucleus. Concomitantly, an increasing percentage of syncytia spontaneously exhibited apoptotic chromatin condensation, as detected with the Hoechst 33342 dye (Figure $1 \mathrm{~A}, \mathrm{~B})$. Karyogamy induced by the ENV-CD4/CXCR4 interaction involved circumscript fusions of the nuclear envelopes and occurred in a fraction of cells which are lacking signs of nuclear chromatin condensation (Figure 1C). It thus may be uncoupled from apoptotic chromatin condensation, as also suggested by kinetic analyses (Figure 1B).

What is then the functional relationship between syncytium formation, karyogamy, and apoptosis? Freshly formed syncytia $(24 \mathrm{~h})$ driven into apoptosis by a shortterm incubation ( $2 \mathrm{~h}$ ) with staurosporin (STS) exhibited a similar degree of chromatin condensation as syncytia undergoing apoptosis spontaneously $(48 \mathrm{~h})$. However neither STS (Figure 1) nor other pro-apoptotic agents such as etoposide (not shown) did increase the frequency of syncytia exhibiting karyogamy, indicating that apoptosis induced by exogenous stimuli obeys other principles than spontaneous syncytial apoptosis. The two pan-caspase inhibitors Boc-D.fmk and Z-VAD.fmk (but not the chemically related cathepsin inhibitor Z-FA.fmk) suppressed nuclear chromatin condensation (Figure $1 A, B)$ as well as the detachment of HeLa Env/HeLa CD4 syncytia from the culture substrate (not shown). Moreover, inhibition of apoptosis by the caspase inhibitor Z-VAD.fmk allowed for the formation of much larger syncytia as compared to control HeLa Env/HeLa CD4 cocultures (Figure 1D), suggesting that caspase activation with the consequent irreversible loss of cellular functions limits the recruitment of cells into growing syncytia.

In strict contrast to their effects on chromatin condensation (Figure 1A,B) and syncytial size (Figure 1D), Boc-D.fmk or Z-VAD.fmk failed to prevent nuclear fusion (Figure 1A,B), suggesting that, karyogamy precedes in a caspase-independent fashion. To further investigate the impact of ongoing fusion events between individual cells/nuclei and existing syncytia (or fusion between syncytia), the monoclonal antibody Leu 3a (which blocks the CDR2 region of the CD4 D1 domain) was added to cocultures. Blockade of CD4 by adding Leu3a from the beginning of cocultures completely abolished cell fusion (Figure 1A). When added after $24 \mathrm{~h}$ of culture, Leu $3 \mathrm{a}$ led to a net reduction of the median number of nuclei per syncytium, strongly inhibited karyogamy (Figure 1A), and, concomitantly, reduced the frequency of apoptotic nuclear events observed $72 \mathrm{~h}$ (Figure 1D) after initiation of coculture.

Altogether, these data suggest that the size of syncytia determines the probability of karyogamy and spontaneous apoptosis and that, inversely, apoptotic caspase activation limits ongoing syncytium formation. More importantly, these data suggest also that both karyogamy and apoptosis may be mechanistically coupled to nonphysiologial syncytium formation. To our knowledge, this is the first description of nuclear fusion in somatic mammalian cells. The molecular mechanisms of nuclear fusion or karyogamy has previously only been explored in the context of sexual reproduction of unicellular fungi such as Saccharomyces cerevisiae. ${ }^{8}$ It will be interesting to learn whether similar mechanisms also participate in cell fusion-induced karyogamy of somatic mammalian cells. 
A

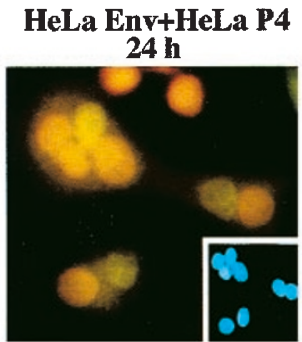

HeLa Env+HeLa P4 $48 \mathrm{~h}$, z.FA.fmk

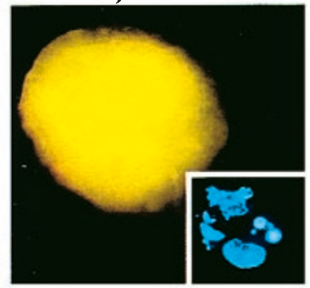

HeLa Env+HeI

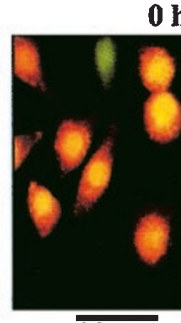

$\overline{20 \mu \mathrm{m}}$

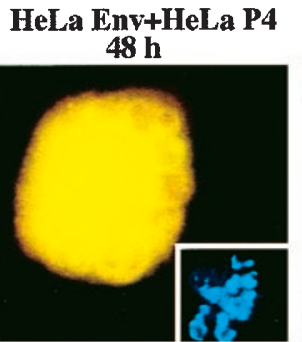

HeLa Env+HeLa P4 $48 \mathrm{~h}$, z.VAD.fmk

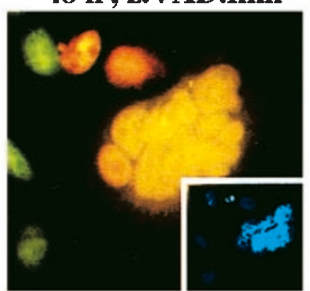

4 HeLa ADA+HeLa P4 $48 \mathrm{~h}$

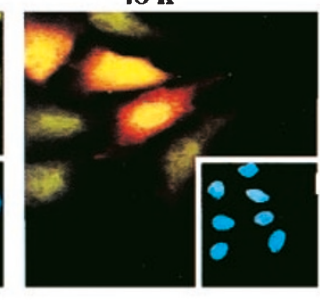

$20 \mu \mathrm{m}$
HeLa Env+HeLa P4

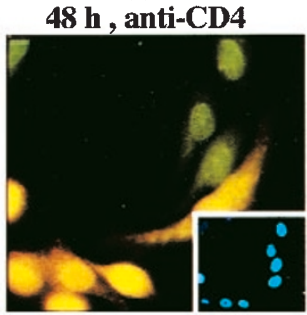

HeLa Env+HeLa P4 $48 \mathrm{~h}$, Boc-D.fmk

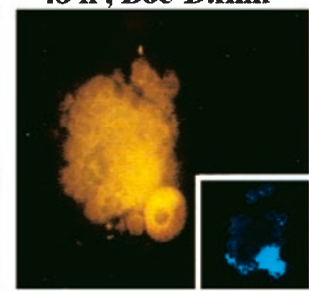

HeLa Env+HeLa P4 $22 \mathrm{~h}$, STS $2 \mathrm{~h}$

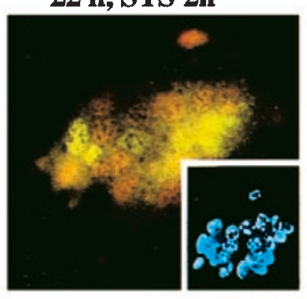

B

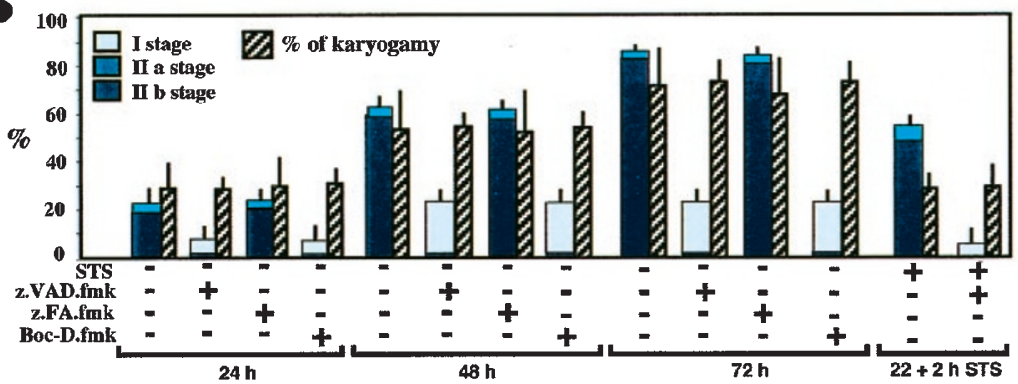

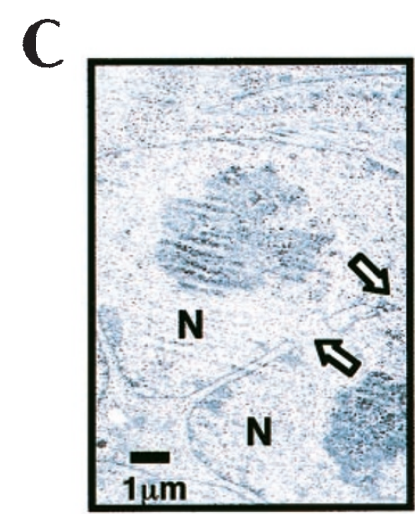

D
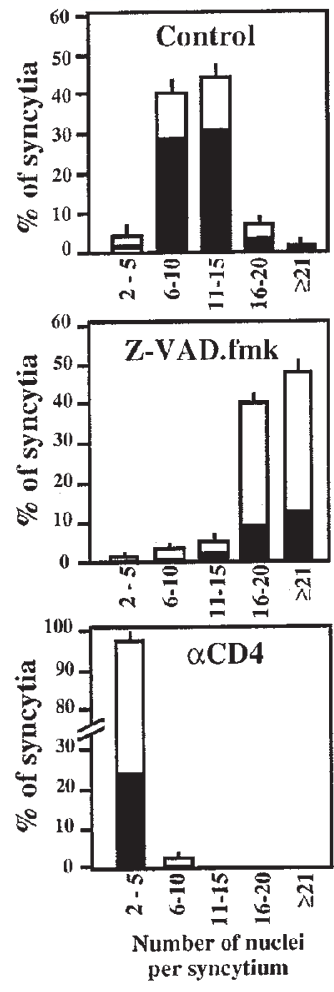

Figure 1 Effect of caspase inhibitors on nuclear apoptosis induced by the interaction between Env and CD4. (A) Morphological evidence for cellular fusion and nuclear chromatin condensation. HeLa Env ${ }^{9}$ (or HeLa ADA cells ${ }^{10}$ were stained with the CellTracker green and co-cultured at a 1:1 ratio with HeLa CD4 (P4) cells stained with CellTracker orange (Molecular Probes, $15 \mu \mathrm{M} ; 30 \mathrm{~min}, 37^{\circ} \mathrm{C}$ ), in the presence or absence of Boc-Asp-fluoromethylketone (Boc-D.fmk), $N$ benzyloxycarbonyl-Val-Ala-Asp-fluoromethylketone (Z-VAD.fmk), N-benzyloxycarbonyl-Phe-Ala-fluoromethylketone (Z-FA.fmk; Enzyme systems; all used at $100 \mu \mathrm{M}$ added each $24 \mathrm{~h}$, or anti-CD4 antibody (Leu3A, $25 \mu \mathrm{g} / \mathrm{ml}$; Becton Dickinson). After the indicated culture period, cells were fixed and counterstained with Hoechst 33324. Staurosporin (STS) was added to 22-hour-old syncytia for $2 \mathrm{~h}$. Blending of the two CellTrackers indicative of nuclear fusion yields a yellow (green+orange) fluorescence. Inserts represent the nuclear morphology obtained with Hoechst 33324 (Sigma; $2 \mu \mathrm{M}, 5 \mathrm{~min}, 37^{\circ} \mathrm{C}$ ). Syncytia representing the dominant phenotype ( $>50 \%$ of cells) are shown. (B) Quantitation of chromatin condensation and nuclear fusion (karyogamy). Cells stained as in A were subjected to a quantitation of the indicated parameters. The stages of chromatin condensation were determined as described. ${ }^{11}$ Results are shown as arithmetic means +S.E.M. ( $n \geqslant 400$ cells) and are representative of three independent experiments. (C) Ultrastructure of nuclear fusion. Arrows denote 'bridges' between nuclei indicative of the fusion of nuclear envelopes leading to blending of the nucleoplasm. This electron micrograph has been obtained from a 24-hour-old syncytium still lacking signs of chromatin condensation. (D) Effect of caspase inhibition and blockade of the CD4/Env interaction on the size and apoptosis of syncytia. HeLa Env cells were cultured with HeLa CD4 cells, in the presence of absence of Z-VAD.fmk (added from the beginning of co-cultures) or CD4 (added at $24 \mathrm{~h}$ ). After $72 \mathrm{~h}$ of co-culture, cells were stained with Hoechst 33324 and the number of nuclei per syncytium, as well as the frequency of nuclear apoptosis (black part of columns), was determined among $\geqslant 600$ cells. This experiment has been repeated twice, yielding similar results 


\section{Acknowledgements}

Supported by a special grant by the Ligue Nationale contre le Cancer, as well as by grants from ANRS, FRM, the European Commission (to G Kroemer). KF Ferri receives a fellowship from the French Ministry of Science. E Jacotot receives an ANRS fellowship. M Geuskens is a senior research associate of the Belgian National Fund for Scientific Research.

\section{KF Ferri ${ }^{1}$, E Jacotot ${ }^{1}, M$ Geuskens $^{2}$ and G Kroemer $^{*}{ }^{* 1}$}

${ }^{1}$ Centre National de la Recherche Scientifique, UMR1599, Institut Gustave Roussy, 39 rue Camille-Desmoulins, F-94805 Villejuif, France; ${ }^{2}$ Laboratory of Molecular Parasitology, Université Libre de Bruxelles, B-6041 Gosselies, Belgium.

*Corresponding author: G Kroemer, CNRS-UMR1599, Institut Gustave Roussy, Pavillon de Recherche 1, 39 rue Camille-Desmoulins, F-94805 Villejuif, France; Tel: 33-1-42 1142 35; Fax: 33-1-42 1152 44;

E-mail: kroemer@infobiogen.fr or kroemer@igr.fr

\author{
1. Sylwester A et al (1997) J. Immunol. 158: 3996-4007 \\ 2. Laurent Crawford AG et al (1993) AIDS Res. Hum. Retroviruses 9: 761-773 \\ 3. Plymale DR et al (1999) AIDS 13: 1827-1839 \\ 4. Amendola A et al (1996) Proc. Natl. Acad. Sci. USA 93: 11057-11062 \\ 5. Ferri KF et al (2000) J. Exp. Med. in press \\ 6. Ferri FK et al (2000) Ann. NY Acad. Sci. in press \\ 7. Dragic T et al (1992) J. Virol. 66: 4794-4802 \\ 8. Rose MD (1996) Annu. Rev. Cell. Dev. Biol. 12: 663-695 \\ 9. Schwartz O et al (1994) Virology 198: 360-365 \\ 10. Pleskoff O et al (1997) Science 276: 1874-1878 \\ 11. Daugas $E$ et al (2000) FASEB. J. 14: 729-739
}

